\title{
On a new fractional Sobolev space with variable exponent on complete manifolds
}

\author{
Ahmed Aberqi' , Omar Benslimane ${ }^{2}$, Abdesslam Ouaziz ${ }^{2}$ and Dušan D. Repovš $\breve{3}^{3 *}$ (I)
}

\section{"Correspondence:}

dusan.repovs@guest.arnes.si

${ }^{3}$ Faculty of Mathematics and

Physics, University of Ljubljana,

Jadranska 19, SI-1000, Ljubljana, Slovenia

Full list of author information is available at the end of the article

\begin{abstract}
We present the theory of a new fractional Sobolev space in complete manifolds with variable exponent. As a result, we investigate some of our new space's qualitative properties, such as completeness, reflexivity, separability, and density. We also show that continuous and compact embedding results are valid. We apply the conclusions of this study to the variational analysis of a class of fractional $p(z, \cdot)$-Laplacian problems involving potentials with vanishing behavior at infinity as an application.
\end{abstract}

Keywords: Fractional $p(z, \cdot)$-Laplacian; Existence of solutions; Fractional Sobolev space with variable exponent on complete manifolds; Variational method

\section{Introduction}

Let $(\mathcal{M}, \mathrm{g})$ be a smooth complete compact Riemannian $n$-manifold. The present paper is devoted to proving some qualitative properties of a new fractional Sobolev space with variable exponent in complete manifolds, as well as to studying the existence of weak solutions to the following problem as an application:

$$
(\mathcal{P})\left\{\begin{array}{l}
\left(-\Delta_{g}\right)_{p(z, \cdot)}^{s} \mathrm{u}(z)+\mathcal{V}(z)|\mathrm{u}(z)|^{q(z)-2} \mathrm{u}=h(z, \mathrm{u}(z)) \text { in } \mathcal{Q}, \\
\left.\mathrm{u}\right|_{\partial \mathcal{Q}}=0,
\end{array}\right.
$$

where $\mathcal{Q} \subset \mathcal{M}$ is an open bounded set with a smooth boundary $\partial \mathcal{Q}, s \in(0,1), p \in C(\mathcal{M} \times$ $\mathcal{M},(1 ; \infty))$ with $s p(z, y)<n$, we assume that $p$ is symmetric and satisfies the following conditions:

$$
\begin{aligned}
& 1<p^{-}=\min _{(z, y) \in \bar{M}^{2}} p(z, y) \leq p(z, y) \leq p^{+}=\max _{(z, y) \in \bar{M}^{2}} p(z, y), \\
& p((z, y)-(x, x))=p(z, y) \quad \forall x, y, z \in M^{3},
\end{aligned}
$$

and we set

$$
\hat{p}(z)=p(z, z), \quad \forall z \in \bar{M},
$$

(c) The Author(s) 2022. This article is licensed under a Creative Commons Attribution 4.0 International License, which permits use, sharing, adaptation, distribution and reproduction in any medium or format, as long as you give appropriate credit to the original author(s) and the source, provide a link to the Creative Commons licence, and indicate if changes were made. The images or other third party material in this article are included in the article's Creative Commons licence, unless indicated otherwise in a credit line to the material. If material is not included in the article's Creative Commons licence and your intended use is not permitted by statutory regulation or exceeds the permitted use, you will need to obtain permission directly from the copyright holder. To view a copy of this licence, visit http://creativecommons.org/licenses/by/4.0/. 
also $q: \overline{\mathcal{M}} \rightarrow(1, \infty)$ satisfies $1<q^{-} \leq q^{+}<p^{-} \leq p^{+}<+\infty$, where $q^{+}=\sup _{z \in \overline{\mathcal{M}}} q(z), q^{-}=$ $\inf _{z \in \overline{\mathcal{M}}} q(z)$, and functions $h, \mathcal{V}$ satisfy some suitable conditions (see Sect. 4).

This type of operator has a significant role in many fields in mathematics, e.g., calculus of variations and partial differential equations, and it has also been used in a variety of physical and engineering contexts, e.g., fluid filtration in porous media, constrained heating, elastoplasticity, image processing, optimal control, financial mathematics, and elsewhere, see $[8,18,37]$ and the references therein.

In recent years, wide research has been done on fractional partial differential equations with variable growth. For example, Bahrouni and Rădulescu [7] developed some qualitative properties on the fractional Sobolev space $W^{s, q(z), p(z, y)}(\mathcal{Q})$ for $s \in(0,1)$ and $\mathcal{Q}$ being a bounded domain in $\mathbb{R}^{n}$ with a Lipschitz boundary. Moreover, they studied the existence of solutions to the following problem:

$$
\begin{cases}\mathcal{L} \mathrm{u}(z)+|\mathrm{u}(z)|^{q(z)-1} \mathrm{u}(z)=\lambda|\mathrm{u}(z)|^{r(z)-1} \mathrm{u}(z) & \text { in } \mathcal{Q}, \\ \mathrm{u}=0 & \text { in } \partial \mathcal{Q}\end{cases}
$$

where

$$
\mathcal{L} \mathrm{u}(z)=p . v . \int_{\mathcal{Q}} \frac{|\mathrm{u}(z)-\mathrm{u}(y)|^{p(z, y)-2}(\mathrm{u}(z)-\mathrm{u}(y))}{|z-y|^{n+s p(z, y)}} d y,
$$

$\lambda>0$, and $1<r(z)<p^{-}=\min _{(z, y) \in \mathcal{Q} \times \mathcal{Q}} p(z, y)$. Bahrouni [6] continued the study of this class of fractional Sobolev spaces with variable exponent and the related nonlocal operator. More precisely, he proved a variant of the comparison principle for $\left(-\Delta_{p(z)}\right)^{s}$. He gave a general principle of sub-supersolution method for the following problem:

$$
\left(\mathcal{P}_{1}\right) \begin{cases}\left(-\Delta_{p(z)}\right)^{s} \mathrm{u}=f(z, \mathrm{u}) & \text { in } \mathcal{Q}, \\ \mathrm{u}=0 & \text { in } \mathbb{R}^{n} \backslash \mathcal{Q},\end{cases}
$$

where $\mathcal{Q}$ is a smooth open bounded domain, $n \geq 3, s \in(0,1), p, f$ are continuous functions, and $f$ satisfies the following assumption:

$$
|f(z, t)| \leq c_{1}+c_{2}|t|^{r(z)-1}, \quad \forall z \in \mathbb{R}^{n}, \forall t \in \mathbb{R}
$$

where $r \in C\left(\mathbb{R}^{n}, \mathbb{R}\right)$ and $1<r(z)<p^{*}(z)=\frac{n p(z, z)}{n-s p(z, z)}, \forall z \in \mathbb{R}^{n}$.

Kaufmann, Rossi, and Vidal [32] proved a compact embedding theorem for fractional Sobolev spaces with variable exponents into variable exponent Lebesgue space and, as an application, they showed the existence and uniqueness of solutions to the following fractional $p(z, y)$-Laplacian equation:

$$
\begin{cases}\mathcal{L} \mathrm{u}(z)+|\mathrm{u}(z)|^{q(z)-2} \mathrm{u}(z)=f(z) & \text { in } \mathcal{Q} \\ \mathrm{u}=0 & \text { in } \partial \mathcal{Q}\end{cases}
$$

with $f \in L^{a(z)}(\mathcal{Q}), a(z)>1$.

In [31] the authors refined the fractional Sobolev spaces with variable exponents given in $[6,7,32]$ and established fundamental embeddings of this space. In addition, they gave 
a sufficient condition for the exponent $p(\cdot, \cdot)$ on $\mathbb{R}^{n} \times \mathbb{R}^{n}$ for the iteration argument of De Giorgi type and proved global boundedness of weak solutions to the problem $\left(\mathcal{P}_{1}\right)$. Readers may refer to $[1,4,5,12-14,19,21-23,27,33,34,36,38,42]$ and the references therein for more ideas and techniques developed to guarantee the existence of weak solutions for a class of nonlocal fractional problems with variable exponents. When $p(\cdot, \cdot)=p=$ constant, we quote, for example, the relevant work of Vázquez [41], see also [2, 9-11, 15, 17, 20, 35] and the references therein. Various techniques have been proposed in the literature in order to recover the compactness in several circumstances. We refer to Tang and Cheng [40], who proposed a new approach to restore the compactness of Palais-Smale sequences, and to Tang and Chen [39], who introduced an original method to recover the compactness of minimizing sequences. A related approach has been developed by Chen and Tang [16] in the framework of Cerami sequences.

Before discussing our main results, we give a review of equations involving the fractional $p$-Laplace operator on Riemannian manifolds. As far as we know, there is only the work of Guo, Zhang, and Zhang [29] who proved the existence of solutions to the following $p$-Laplacian equations with homogeneous Dirichlet boundary conditions:

$$
\begin{cases}\left(-\Delta_{g}\right)_{p}^{s} \mathrm{u}(z)=f(z, \mathrm{u}(x)) & \text { in } \mathcal{Q}, \\ \mathrm{u}=0 & \text { in } M \backslash \mathcal{Q},\end{cases}
$$

where $s p<n$ with $s \in(0,1), p \in(1 ; \infty),\left(-\Delta_{g}\right)_{p}^{s}$ is the fractional $p$-Laplacian on Riemannian manifolds, $(M, g)$ is a compact Riemannian $n$-manifold, $\mathcal{Q}$ is an open bounded subset of $M$ with a smooth boundary $\partial \mathcal{Q}$, and $f$ is a Carathéodory function satisfying the AmbrosettiRabinowitz-type condition.

The motivation of this paper was, on the one hand, the work of Fu and Guo [24] who introduced the variable exponent function spaces on Riemannian manifolds in 2012, followed by Gaczkowski and Górka [25] who in 2013 examined the above space in the case of compact manifolds, and Guo [28] who in 2015 discussed the properties of the Nemytsky operator and obtained the existence of weak solutions for Dirichlet problems of nonhomogeneous $p(m)$-harmonic equations. Finally, in 2016 Gaczkowski, Górka, and Pons [26] studied the variable exponent function spaces on complete noncompact Riemannian manifolds. Furthermore, they proved the continuous embeddings results between Sobolev and Hölder function spaces, using classic assumptions on the geometry. In addition, they established the compact embeddings of $H$-invariant Sobolev spaces, where $H$ is a compact Lie subgroup of the manifold group of isometries, and, as an application, they showed the existence of weak solutions to nonhomogeneous $q(z)$-Laplace equations. For further background, we recommend that readers consult $[1,12]$ and the references therein. On the other hand, we were also motivated by the work of Guo, Zhang, and Zhang [29] who established the theory of fractional Sobolev spaces on Riemannian manifolds.

The novelty of our work is in extending Sobolev spaces with variable exponents to cover the fractional case with complete manifolds. We prove some qualitative properties of this new space. Next, we study the existence of solutions to some nonlocal problems involving potentials allowed for vanishing behavior at infinity. However, the main difficulty is presented by the fact that the $p(z)$-Laplacian operator has a more complicated nonlinearity than the $p$-Laplacian operator. For example, it is nonhomogeneous. To the best of our knowledge, there is no known result along this line. 
The outline of the paper is as follows. In Sect. 2, we collect the pertinent properties and notations of Lebesgue spaces with variable exponents and Sobolev-Orlicz spaces with variable exponents on a complete manifold. Moreover, we show the relation between the norm and the modular. In Sect. 3, we study the completeness, reflexivity, separability, and density of our new space. Furthermore, we prove a continuous and compact embedding theorem of this space into variable exponent Lebesgue spaces. In Sect. 4, we deal with a fractional $p(z)$-Laplacian problem involving potentials allowed for vanishing behavior at infinity as an application.

\section{Preliminaries}

In this section, we review some definitions and properties of spaces $W_{0}^{1, q(z)}(\mathcal{Q})$, where $\mathcal{Q}$ is an open subset of $\mathbb{R}^{n}$, and $W_{0}^{1, q(z)}(\mathcal{M})$, which are known as the Sobolev spaces with variable exponents and the Sobolev spaces with variable exponents on a complete manifold, respectively. For more background, we refer to $[1,3,12,21,26,28,30]$ and the references therein.

\subsection{Sobolev spaces with variable exponents}

Suppose that $\mathcal{Q} \subset \mathbb{R}^{n}$ is a bounded open domain, with $n \geq 2$. Let $q(\cdot): \mathcal{Q} \rightarrow(1, \infty)$ be a measurable function. We define real numbers $q^{+}$and $q^{-}$as follows:

$$
q^{+}=\operatorname{ess} \sup \{q(z): z \in \mathcal{Q}\} \quad \text { and } \quad q^{-}=\operatorname{essinf}\{q(z): z \in \mathcal{Q}\}
$$

Definition 2.1 ([21]) We define the Lebesgue space with variable exponent $L^{q(\cdot)}(\mathcal{Q})$ as follows:

$$
L^{q(\cdot)}(\mathcal{Q})=\left\{\mathrm{u}: \mathcal{Q} \rightarrow \mathbb{R}: \varrho_{q(\cdot)}(\mathrm{u})=\int_{\mathcal{Q}}|\mathrm{u}(z)|^{q(z)} d z<+\infty\right\}
$$

and endow it with the Luxemburg norm

$$
\|\mathrm{u}\|_{L^{q(\cdot)}(\mathcal{Q})}=\inf \left\{\mu>0: \varrho_{q(\cdot)}\left(\frac{\mathrm{u}}{\mu}\right) \leq 1\right\}
$$

if $q^{+}<+\infty$.

Proposition $2.1([21])\left(L^{q(\cdot)}(\mathcal{Q}),\|\cdot\|_{L^{q \cdot(\cdot)}(\mathcal{Q})}\right)$ is a separable Banach space, and uniformly convex for $1<q^{-} \leq q^{+}<+\infty$, hence reflexive.

Proposition 2.2 (Hölder inequality, [21])

$$
\left|\int_{\mathcal{Q}} \mathrm{u} v d x\right| \leq\left(\frac{1}{q^{-}}+\frac{1}{\left(q^{\prime}\right)^{-}}\right)\|\mathrm{u}\|_{L^{q^{(\cdot)}(\mathcal{Q})}}\|v\|_{L^{q^{\prime}(\cdot)}(\mathcal{Q})}, \quad \forall \mathrm{u}, v \in L^{q(\cdot)}(\mathcal{Q}) \times L^{q^{\prime(\cdot)}(\mathcal{Q})}
$$

with $\frac{1}{q(z)}+\frac{1}{q^{\prime}(z)}=1$.

Definition 2.2 ([21]) We define the variable exponent Sobolev space by

$$
W^{1, q(z)}(\mathcal{Q})=\left\{\mathrm{u}: \mathrm{u} \in L^{q(z)}(\mathcal{Q}) \text { and }|D \mathrm{u}| \in L^{q(z)}(\mathcal{Q})\right\}
$$


end endow it with the norm

$$
\|\mathrm{u}\|_{W^{1, q(z)}(\mathcal{Q})}=\|\mathrm{u}\|_{L^{q(z)}(\mathcal{Q})}+\|D \mathrm{u}\|_{L^{q(z)}(\mathcal{Q})}, \quad \forall \mathrm{u} \in W^{1, q(z)}(\mathcal{Q})
$$

and set $W_{0}^{1, q(z)}(\mathcal{Q}):={\overline{C_{0}^{\infty}(\mathcal{Q})}}^{W^{1, q(z)}(\mathcal{Q})}$.

\subsection{Sobolev spaces with variable exponents on complete manifolds}

Let $(\mathcal{M}, \mathrm{g})$ be a smooth complete compact Riemannian $n$-manifold. We begin by recalling some background, more can be found in $[1,3,26,28,30]$. A chart of manifold $\mathcal{M}$ is a couple $(\mathcal{Q}, \varphi)$, where $\varphi$ is a homeomorphism of the open set $\mathcal{Q}$ onto some open subset of $\mathbb{R}^{n}$. Furthermore, a collection of charts $\left(\mathcal{Q}_{i}, \varphi_{i}\right)_{i \in I}$ such that $\mathcal{M}=\bigcup_{i \in I} \mathcal{Q}_{i}$ is called an atlas on $\mathcal{M}$

Remark 2.1 ([30, page 9]) For any atlas $\left(\mathcal{Q}_{i}, \varphi_{i}\right)_{i \in I}$ on $\mathcal{M}$, there exists a partition of unity $\left(\mathcal{Q}_{j}, \varphi_{j}, \eta_{j}\right)_{j \in J}$ subordinate to the covering $\left(\mathcal{Q}_{i}\right)_{i \in I}$.

Now, we define a natural positive Radon measure.

Definition 2.3 ([30, page 9)]) Let $\mathrm{u}: \mathcal{M} \rightarrow \mathbb{R}$ be continuous with compact support, and let $\left(\mathcal{Q}_{i}, \varphi_{i}\right)_{i \in I}$ be an atlas on $\mathcal{M}$, and set

$$
\int_{\mathcal{M}} \mathrm{u}(z) d v_{\mathrm{g}}(z)=\sum_{k \in J} \int_{\varphi_{k}\left(\mathcal{Q}_{k}\right)}\left(\left(\operatorname{det}\left(\mathrm{g}_{i j}\right)\right)^{\frac{1}{2}} \eta_{k} \mathrm{u}\right) o \varphi_{k}^{-1}(z) d z,
$$

where $d v_{\mathrm{g}}=\left(\operatorname{det}\left(\mathrm{g}_{i j}\right)\right)^{\frac{1}{2}} d z$ is the Riemannian volume element on $(\mathcal{M}, \mathrm{g}), \mathrm{g}_{i j}$ are the components of the Riemannian metric $\mathrm{g}$ in the chart, and $d z$ is the Lebesgue volume element of $\mathbb{R}^{n}$.

Next, we define the Sobolev spaces $L_{k}^{q(\cdot)}(\mathcal{M})$ as the completion of $C_{k}^{q(\cdot)}(\mathcal{M})$ with respect to the norm $\|\mathrm{u}\|_{L_{k}^{q(\cdot)}}$, where

$$
C_{k}^{q(\cdot)}(\mathcal{M})=\left\{\mathrm{u} \in C^{\infty}(\mathcal{M}) \text { such that } \forall j, 0 \leq j \leq k,\left|D^{k} \mathrm{u}\right| \in L^{q(\cdot)}(\mathcal{M})\right\}
$$

and

$$
\|\mathrm{u}\|_{L_{k}^{q(\cdot)}}=\sum_{j=0}^{k}\left\|D^{j} \mathrm{u}\right\|_{L^{q(\cdot)}},
$$

with $\left|D^{k} \mathrm{u}\right|$ being the norm of the $k$ th covariant derivative of $\mathrm{u}$, defined in local coordinates by

$$
\left|D^{k} \mathrm{u}\right|^{2}=\mathrm{g}^{i_{1} j_{1}} \cdots \mathrm{g}^{i_{k} j_{k}}\left(D^{k} \mathrm{u}\right)_{i_{1} \ldots i_{k}}\left(D^{k} \mathrm{u}\right)_{j_{1} \ldots j_{k}}
$$

Definition 2.4 ([3]) Let $\zeta:[\alpha, \beta] \rightarrow \mathcal{M}$ be a curve of class $C^{1}$. The length of $\zeta$ is

$$
\ell(\zeta)=\int_{\alpha}^{\beta} \sqrt{\mathrm{g}\left(\frac{d \gamma}{d s}, \frac{d \gamma}{d s}\right)} d s,
$$


and, for a pair of points $z, y \in \mathcal{M}$, we define the distance $d_{\mathrm{g}}(z, y)$ between $z$ and $y$ by

$$
d_{\mathrm{g}}(z, y)=\inf \{\ell(\zeta): \zeta[\alpha, \beta] \rightarrow \mathcal{M} \text { such as } \zeta(\alpha)=z \text { and } \zeta(\beta)=y\}
$$

Definition 2.5 ([26]) A function $t: \mathcal{M} \rightarrow \mathbb{R}$ is log-Hölder continuous if there exists a constant $C$ such that, for every pair of points $\{z, y\}$ in $\mathcal{M}$,

$$
|t(z)-t(y)| \leq C\left(\log \left(e+\frac{1}{d_{\mathrm{g}}(z, y)}\right)\right)^{-1} .
$$

Let $\mathcal{P}^{\log }(\mathcal{M})$ be the set of $\log$-Hölder continuous real functions on $\mathcal{M}$, which is linked to $\mathcal{P}^{\log }\left(\mathbb{R}^{n}\right)$ by the following proposition:

Proposition $2.3([3,26])$ Given $q \in \mathcal{P}^{\log }(\mathcal{M})$, let $(\mathcal{Q}, \phi)$ be a chart such that

$$
\frac{1}{2} \delta_{i j} \leq \mathrm{g}_{i j} \leq 2 \delta_{i j}
$$

as bilinear forms, where $\delta_{i j}$ is the Kronecker delta symbol. Then $q \circ \phi^{-1} \in \mathcal{P}^{\log }(\phi(\mathcal{Q}))$.

Definition 2.6 ([3]) If the Ricci tensor of $\mathrm{g}$, denoted by $R c(\mathrm{~g})$, satisfies $R c(\mathrm{~g}) \geq \lambda(n-1) \mathrm{g}$, for some $\lambda$ and for all $z \in \mathcal{M}, \exists v>0$ such that $\left|B_{1}(z)\right|_{\mathrm{g}} \geq v$, where $B_{1}(z)$ are balls of radius 1 centered at some point $z$ in terms of the volume of smaller concentric balls, then we say that the $n$-manifold $(\mathcal{M}, \mathrm{g})$ has property $B_{v o l}(\lambda, v)$.

Proposition 2.4 ([1, Proposition 2.17]) Let $\mathrm{u} \in L^{q(z)}(\mathcal{M}),\left\{\mathrm{u}_{k}\right\}_{k \geq 0} \subset L^{q(z)}(\mathcal{M})$. Then

(i) $\|\mathrm{u}\|_{L^{q(z)}(\mathcal{M})}<1 \Longrightarrow\|\mathrm{u}\|_{L^{q(z)}(\mathcal{M})}^{q^{+}} \leq \varrho_{q(z)}(\mathrm{u}) \leq\|\mathrm{u}\|_{L^{q(z)}(\mathcal{M})}^{q^{-}}$,

(ii) $\|\mathrm{u}\|_{L^{q(z)}(\mathcal{M})}>1 \Longrightarrow\|\mathrm{u}\|_{L^{q(z)}(\mathcal{M})}^{q^{-}} \leq \varrho_{q(z)}(\mathrm{u}) \leq\|\mathrm{u}\|_{L^{q(z)}(\mathcal{M})}^{q^{+}}$, where

$$
\varrho_{q(z)}(\mathrm{u})=\int_{\mathcal{M}}|\mathrm{u}(z)|^{q(z)} d v_{g}(z) .
$$

We now prove the following proposition.

Proposition 2.5 If $\mathrm{u}, \mathrm{u}_{k} \in L^{q(z)}(\mathcal{M})$ and $k \in \mathbb{N}$, then the following assertions are equivalent:

(1) $\lim _{k \rightarrow+\infty}\left\|u_{k}-u\right\|_{L^{q(z)}(\mathcal{M})}=0$,

(2) $\lim _{k \rightarrow+\infty} \varrho_{q(z)}\left(\mathrm{u}_{k}-\mathrm{u}\right)=0$,

(3) $\mathrm{u}_{k} \rightarrow \mathrm{u}$ a.e. on $\mathcal{M}$ and $\lim _{k \rightarrow+\infty} \varrho_{q(\cdot)}\left(\mathrm{u}_{k}\right)=\varrho_{q(\cdot)}(\mathrm{u})$.

Proof If $\left\|\mathrm{u}_{k}-\mathrm{u}\right\|_{L^{q(z)}(\mathcal{M})} \rightarrow 0$, then

$$
\lim _{k \rightarrow+\infty} \int_{\mathcal{M}}\left|\mathrm{u}_{k}-\mathrm{u}\right|^{q(z)} d v_{\mathrm{g}}(z)=0 .
$$

It is now easy to observe that $\mathrm{u}_{k} \rightarrow \mathrm{u}$ a.e. on $\mathcal{M}$. Thus $\left|\mathrm{u}_{k}\right|^{q(z)} \rightarrow|\mathrm{u}|^{q(z)}$ on $\mathcal{M}$ and the integrals of the functions $\left|\mathrm{u}_{k}-\mathrm{u}\right|^{q(z)}$ are absolutely equicontinuous on $\mathcal{M}$, and since

$$
\left|\mathbf{u}_{k}\right|^{q(z)} \leq 2^{q^{+}-1}\left(\left|\mathbf{u}_{k}-\mathrm{u}\right|^{q(z)}+|\mathrm{u}|^{q(z)}\right),
$$


the integrals of the $\left|\mathrm{u}_{k}\right|^{q(z)}$ are also absolutely equicontinuous on $\mathcal{M}$, so, by the Vitali convergence theorem, we obtain that

$$
\lim _{k \rightarrow+\infty} \varrho_{q(\cdot)}\left(\mathrm{u}_{k}\right)=\varrho_{q(\cdot)}(\mathrm{u})
$$

Conversely, if $\mathrm{u}_{k} \rightarrow \mathrm{u}$ on $\mathcal{M}$, we can deduce that $\left|\mathrm{u}_{k}-\mathrm{u}\right|^{q(z)} \rightarrow 0$ on $\mathcal{M}$, and using the same techniques as in the above proof, and due to the fact that

$$
\left|\mathbf{u}_{k}-\mathrm{u}\right|^{q(z)} \leq 2^{q^{+}-1}\left(\left|\mathbf{u}_{k}\right|^{q(z)}+|\mathrm{u}|^{q(z)}\right)
$$

and $\lim _{k \rightarrow+\infty} \varrho_{q(\cdot)}\left(\mathrm{u}_{k}\right)=\varrho_{q(\cdot)}(\mathrm{u})$, we obtain that $\lim _{k \rightarrow+\infty} \varrho_{q(\cdot)}\left(\mathrm{u}_{k}-\mathrm{u}\right)=0$.

Remark 2.2 The following relation will be used to compare the functionals $\|\cdot\|_{L^{q(\cdot)}(\mathcal{M})}$ and $\varrho_{q(\cdot)}(\cdot):$

$$
\min \left\{\varrho_{q(\cdot)}(\mathrm{u})^{\frac{1}{q^{-}}}, \varrho_{q(\cdot)}(\mathrm{u})^{\frac{1}{q^{+}}}\right\} \leq\|\mathrm{u}\|_{L^{q(\cdot)}(\mathcal{M})} \leq \max \left\{\varrho_{q(\cdot)}(\mathrm{u})^{\frac{1}{q^{-}}}, \varrho_{q(\cdot)}(\mathrm{u})^{\frac{1}{q^{+}}}\right\} .
$$

Definition 2.7 ([28]) The Sobolev space $W^{1, q(z)}(\mathcal{M})$ consists of all functions $\mathrm{u} \in L^{q(z)}(\mathcal{M})$ for which $D^{k} \mathrm{u} \in L^{q(z)}(\mathcal{M}) k=1,2, \ldots, n$. The norm is defined by

$$
\|\mathrm{u}\|_{W^{1, q(z)}(\mathcal{M})}=\|\mathrm{u}\|_{L^{q(z)}(\mathcal{M})}+\sum_{k=1}^{n}\left\|D^{k} u\right\|_{L^{q(z)}(\mathcal{M})} .
$$

The space $W_{0}^{1, q(z)}(\mathcal{M})$ is defined as the closure of $C^{\infty}(\mathcal{M})$ in $W^{1, q(z)}(\mathcal{M})$.

Theorem 2.1 ([1]) Let $\mathcal{M}$ be a compact Riemannian manifold with a smooth boundary or without boundary and $q(z), p(z) \in C(\overline{\mathcal{M}}) \cap L^{\infty}(\mathcal{M})$. Assume that

$$
q(z)<n, \quad p(z)<\frac{n q(z)}{n-q(z)} \quad \text { for } z \in \overline{\mathcal{M}} .
$$

Then

$$
W^{1, q(z)}(\mathcal{M}) \hookrightarrow L^{p(z)}(\mathcal{M})
$$

is a continuous and compact embedding.

Proposition 2.6 ([3]) If $(\mathcal{M}, \mathrm{g})$ is complete, then $W^{1, q(z)}(\mathcal{M})=W_{0}^{1, q(z)}(\mathcal{M})$.

\section{Fractional Sobolev space with variable exponent on a complete manifold}

On a complete manifold, we introduce in this section a new fractional Sobolev space with variable exponent and state our mains results.

Definition 3.1 Let $p: \overline{\mathcal{M}} \times \overline{\mathcal{M}} \rightarrow(1 ; \infty)$ be a continuous variable exponent and let $s \in$ $(0,1)$. We define the modular

$$
\varrho_{p(\cdot, \cdot)}(\mathrm{u})=\int_{\mathcal{M} \times \mathcal{M}} \frac{|\mathrm{u}(z)-\mathrm{u}(y)|^{p(z, y)}}{\left(d_{\mathrm{g}}(z, y)\right)^{n+s p(z, y)}} d v_{\mathrm{g}}(z) d v_{\mathrm{g}}(y)
$$


For $s \in(0,1)$, we introduce the variable exponent Sobolev fractional space on a complete manifold as follows:

$$
\begin{aligned}
W^{s, p(z, y)}(\mathcal{M})= & \left\{\mathrm{u}: \mathcal{M} \rightarrow \mathbb{R}: \mathrm{u} \in L^{\hat{p}(z)}(\mathcal{M})\right. \text { such as } \\
& \left.\int_{\mathcal{M} \times \mathcal{M}} \frac{|\mathrm{u}(z)-\mathrm{u}(y)|^{p(z, y)}}{\left(d_{\mathrm{g}}(z, y)\right)^{n+s p(z, y)}} d v_{\mathrm{g}}(z) d v_{\mathrm{g}}(y)<\infty \text {, for some } \lambda>0\right\} .
\end{aligned}
$$

Consequently,

$$
\|\mathrm{u}\|_{\varrho_{p(, \cdot)}}=\inf \left\{\lambda>0: \varrho_{p(\cdot,)}\left(\frac{\mathrm{u}}{\lambda}\right) \leq 1\right\}=[\mathrm{u}]_{W^{s, p(z, y)}(\mathcal{M})} .
$$

The modular $\varrho_{p(\cdot,)}$ has the following properties.

\subsection{Lemmas}

In this part, we will go through some of our new fractional space's qualitative lemmas.

Lemma 3.1 Let $p \in C(\mathcal{M} \times \mathcal{M},(1 ; \infty))$ be a continuous variable exponent. Then for any $\mathrm{u} \in W^{s, p(z, y)}(\mathcal{M})$, we get

(1) $[\mathrm{u}]_{W^{s, p(z, y)}(\mathcal{M})} \geq 1 \Longrightarrow[\mathrm{u}]_{W^{s, p(z, y)}(\mathcal{M})}^{p^{-}} \leq \varrho_{p(\cdot, \cdot)}(\mathrm{u}) \leq[\mathrm{u}]_{W^{s, p(z, y)}(\mathcal{M})^{p^{+}}}$,

(2) $[\mathrm{u}]_{W^{s, p(z, y)}(\mathcal{M})} \leq 1 \Longrightarrow[\mathrm{u}]_{W^{p^{+}, p(z, y)}(\mathcal{M})} \leq \varrho_{p(\cdot, \cdot)}(\mathrm{u}) \leq[\mathrm{u}]_{W^{s, p(z, y)}(\mathcal{M})}^{p^{-}}$.

Proof (1) For all $\theta \in(0,1)$, we have

$$
\theta^{p^{+}} \varrho_{p(\cdot,)}(\mathrm{u}) \leq \varrho_{p(\cdot,)}(\theta \mathrm{u}) \leq \theta^{p^{-}} \varrho_{p(\cdot, \cdot)}(\mathrm{u})
$$

So, if $[\mathrm{u}]_{W^{s, p(z, y)}(\mathcal{M})}>1$, then $0<\frac{1}{\left[\mathrm{u}_{W^{s, p}(z, y)}(\mathcal{M})\right.}<1$, thus we have

$$
\frac{\varrho_{p(\cdot,)}(\mathrm{u})}{[\mathrm{u}]_{W^{s, p(p, y)}(\mathcal{M})}^{p^{+}}} \leq \varrho_{p(\cdot,)}\left(\frac{\mathrm{u}}{[\mathrm{u}]_{W^{s, p(z, y)}(\mathcal{M})}}\right) \leq \frac{\varrho_{p(\cdot,)}(\mathrm{u})}{[\mathrm{u}]_{W^{s, p},(z, y)}^{p^{-}(\mathcal{M})}},
$$

and, since $\varrho_{p(\cdot, \cdot)}\left(\frac{u}{[u]_{W}, p(z, y)(\mathcal{M})}\right)=1$, obtain our result. We proceed in the same way for (2).

Remark 3.1 It is important to note that the results of Proposition 2.5 apply to $\varrho_{p(, .)}$.

Lemma 3.2 If $(\mathcal{M}, \mathrm{g})$ be a smooth complete compact Riemannian n-manifold, then $W^{s, p(z, y)}(\mathcal{M})$ is a Banach space.

Proof Let $\left\{\mathrm{u}_{n}\right\}$ be a Cauchy sequence in $W^{s, p(z, y)}(\mathcal{M})$. Since

$$
\hat{p}(z)<\hat{p}_{s}^{*}(z)= \begin{cases}\frac{n \hat{p}(z)}{n-s \hat{p}(z)} & \text { if } s \hat{p}(z)<n \\ +\infty & \text { otherwise }\end{cases}
$$

for any $z \in \overline{\mathcal{M}}$, it follows that for any $\eta>0$, there exists $\mu_{\eta}$ such that, if $\ell, m \geq \mu_{\eta}$,

$$
\left\|\mathrm{u}_{\ell}-\mathrm{u}_{m}\right\|_{L^{\hat{p}(z)}(\mathcal{M})} \leq\left\|\mathrm{u}_{\ell}-\mathrm{u}_{m}\right\|_{W^{s, p(z, y)}(\mathcal{M})} \leq \eta .
$$


Since $L^{\hat{p}(z)}(\mathcal{M})$ is complete (Lemma 2.5 in [28]), there exists $\mathrm{u} \in L^{\hat{p}(z)}(\mathcal{M})$ such that $\mathrm{u}_{\ell} \rightarrow \mathrm{u}$ strongly in $L^{\hat{p}(z)}(\mathcal{M})$ as $\ell \rightarrow+\infty$. Consequently, we may find a subsequence $\left\{u_{\ell_{t}}\right\}$ of $\left\{u_{\ell}\right\}$ in $W^{s, p(z, y)}(\mathcal{M})$ such that $\mathrm{u}_{\ell_{t}} \rightarrow$ u a.e. on $\mathcal{M}$.

Then, by the Fatou's lemma and (3) with $\eta=1$, we obtain

$$
\begin{aligned}
& \varrho_{p(\cdot,)}(\mathrm{u}) \\
& =\int_{\mathcal{M} \times \mathcal{M}} \frac{|\mathrm{u}(z)-\mathrm{u}(y)|^{p(z, y)}}{\left(d_{\mathrm{g}}(z, y)\right)^{n+s p(z, y)}} d v_{\mathrm{g}}(z) d v_{\mathrm{g}}(y) \\
& \leq \lim _{t \rightarrow+\infty} \inf \int_{\mathcal{M} \times \mathcal{M}} \frac{\left|\mathbf{u}_{\ell_{t}}(z)-\mathrm{u}_{\ell_{t}}(y)\right|^{p(z, y)}}{\left(d_{\mathrm{g}}(z, y)\right)^{n+s p(z, y)}} d v_{\mathrm{g}}(z) d v_{\mathrm{g}}(y) \\
& \leq 2^{p^{+}-1} \lim _{t \rightarrow+\infty} \inf \left[\int_{\mathcal{M} \times \mathcal{M}} \frac{\left|\left(\mathrm{u}_{\ell_{t}}(z)-\mathrm{u}_{\mu_{1}}(z)\right)-\left(\mathrm{u}_{\ell_{t}}(y)-\mathrm{u}_{\mu_{1}}(z)\right)\right|^{p(z, y)}}{\left(d_{\mathrm{g}}(z, y)\right)^{n+s p(z, y)}} d v_{\mathrm{g}}(z) d v_{\mathrm{g}}(y)\right. \\
& \left.+\int_{\mathcal{M} \times \mathcal{M}} \frac{\left|\mathrm{u}_{\mu_{1}}(z)-\mathrm{u}_{\mu_{1}}(y)\right|^{p(z, y)}}{\left(d_{\mathrm{g}}(z, y)\right)^{n+s p(z, y)}} d v_{\mathrm{g}}(z) d v_{g}(y)\right] \\
& \leq 2^{p^{+}-1}\left[\lim _{t \rightarrow+\infty} \inf \varrho_{p(\cdot, \cdot)}\left(\mathrm{u}_{\ell_{t}}-\mathrm{u}_{\mu_{1}}\right)+\varrho_{p(\cdot,)}\left(\mathrm{u}_{\mu_{1}}\right)\right]
\end{aligned}
$$

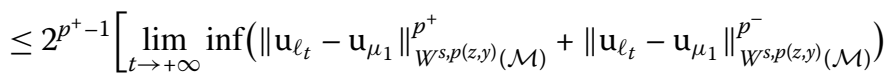

$$
\begin{aligned}
& \left.+\left(\left\|\mathrm{u}_{\mu_{1}}\right\|_{W^{s, p(z, y)}(\mathcal{M})}^{p^{+}}+\left\|\mathrm{u}_{\mu_{1}}\right\|_{W^{s, p(z, y)}(\mathcal{M})}^{p^{-}}\right)\right] \\
& \leq 2^{p^{+}-1}\left(2+\left\|\mathrm{u}_{\mu_{1}}\right\|_{W^{s, p(z, y)}(\mathcal{M})}^{p^{+}}+\left\|\mathrm{u}_{\mu_{1}}\right\|_{W^{s, p(z, y)}(\mathcal{M})}^{p^{-}}\right)<+\infty .
\end{aligned}
$$

Hence, $\mathrm{u} \in W^{s, p(z, y)}(\mathcal{M})$. On the other hand, let $\ell \geq \mu_{\eta}$. Then, according to (3) and from Fatou's lemma, we get

$$
\varrho_{p(\cdot,)}\left(\mathrm{u}_{\ell}-\mathrm{u}\right) \leq \lim _{t \rightarrow+\infty} \inf \varrho_{p(\cdot,)}\left(\mathrm{u}_{\ell}-\mathrm{u}_{\ell}\right) \leq \frac{\eta^{p^{+}}+\eta^{p^{-}}}{2}=\eta^{*} .
$$

Thus $\lim _{\ell \rightarrow+\infty} \varrho_{p(\cdot, \cdot)}\left(\mathrm{u}_{\ell}-\mathrm{u}\right)=0$. Thanks to Remark 3.1, $\lim _{\ell \rightarrow+\infty}\left\|\mathrm{u}_{\ell}-\mathrm{u}\right\|_{W^{s, p(z, y)}(\mathcal{M})}=0$. That is, $\mathrm{u}_{\ell} \rightarrow \mathrm{u}$ strongly on $W^{s, p(z, y)}(\mathcal{M})$ as $\ell \rightarrow+\infty$.

Lemma 3.3 Let $(\mathcal{M}, \mathrm{g})$ be a smooth complete compact Riemannian n-manifold, and $p(z, y) \in C(\mathcal{M} \times \mathcal{M},(1, \infty))$ with $s p(z, y)<n$, for $z, y \in \overline{\mathcal{M}}$. Then $W^{s, p(z, y)}(\mathcal{M})$ is a separable and reflexive space.

Proof Consider $\mathrm{u}, v \in W_{0}^{s, p(z, y)}(\mathcal{M})$ satisfying $\|\mathrm{u}\|_{W_{0}^{s, p(z, y)}(\mathcal{M})}=\|v\|_{W_{0}^{s, p(z, y)}(\mathcal{M})}=1$ and $\| \mathrm{u}-$ $v \|_{W_{0}^{s, p(z, y)}(\mathcal{M})} \geq \varepsilon$, where $\varepsilon \in(0,2)$.

Case $p(z, y) \geq 2$. By inequality (28) in [2], we have that

$$
\begin{aligned}
& \left\|\frac{\mathrm{u}+v}{2}\right\|_{W_{0}^{s, p(z, y)}(\mathcal{M})}^{p(z, y)}+\left\|\frac{\mathrm{u}-v}{2}\right\|_{W_{0}^{s, p(z, y)}(\mathcal{M})}^{p(z, y)} \\
& \leq\left(\frac{1}{2}\right)^{\frac{p^{+}}{p-}} \int_{\mathcal{M} \times \mathcal{M}} \frac{|\mathrm{u}(z)-\mathrm{u}(y)|^{p(z, y)}}{\left(d_{\mathrm{g}}(z, y)\right)^{n+s p(z, y)}} d v_{\mathrm{g}}(z) d v_{\mathrm{g}}(y) \\
& \quad+\left(\frac{1}{2}\right)^{\frac{p^{+}}{p}} \int_{\mathcal{M} \times \mathcal{M}} \frac{|v(z)-v(y)|^{p(z, y)}}{\left(d_{\mathrm{g}}(z, y)\right)^{n+s p(z, y)}} d v_{\mathrm{g}}(z) d v_{\mathrm{g}}(y)
\end{aligned}
$$




$$
\begin{aligned}
& <\frac{1}{2} \int_{\mathcal{M} \times \mathcal{M}} \frac{|\mathrm{u}(z)-\mathrm{u}(y)|^{p(z, y)}}{\left(d_{\mathrm{g}}(z, y)\right)^{n+s p(z, y)}} d \nu_{\mathrm{g}}(z) d \nu_{\mathrm{g}}(y) \\
& \quad+\frac{1}{2} \int_{\mathcal{M} \times \mathcal{M}} \frac{|v(z)-v(y)|^{p(z, y)}}{\left(d_{\mathrm{g}}(z, y)\right)^{n+s p(z, y)}} d \nu_{\mathrm{g}}(z) d \nu_{\mathrm{g}}(y) \\
& =\frac{1}{2}\|\mathrm{u}\|_{W_{0}^{s, p(z, y)}(\mathcal{M})}^{p(z, y)}+\frac{1}{2}\|v\|_{W_{0}^{s, p p(z, y)}(\mathcal{M})}^{p(z, y)}=1 .
\end{aligned}
$$

So, $\left\|\frac{\mathrm{u}+v}{2}\right\|_{W_{0}^{s, p(z, y)}(\mathcal{M})}^{p(z, y)} \leq 1-(\varepsilon / 2)^{p(z, y)}$. Taking $\delta=\delta(\varepsilon)$ such that $1-(\varepsilon / 2)^{p(z, y)}=(1-\delta)^{p(z, y)}$, we obtain $\left\|\frac{\mathrm{u}+v}{2}\right\|_{W_{0}^{s, p(z, y)}(\mathcal{M})} \leq(1-\delta)$.

Case $1<p(z, y)<2$. Letting $p^{\prime}(z, y)=\frac{p(z, y)}{(p(z, y)-1)}$, we have

$$
\|\mathrm{u}\|_{W_{0}^{s, p(z, y)}(\mathcal{M})}^{p^{\prime}(z, y)}=\left[\int_{\mathcal{M} \times \mathcal{M}}\left(\left(\frac{|\mathrm{u}(z)-\mathrm{u}(y)|}{\left(d_{\mathrm{g}}(z, y)\right)^{\frac{n}{p(z, y)}}+s}\right)^{p^{\prime}(z, y)}\right)^{p(z, y)-1} d \nu_{\mathrm{g}}(z) d \nu_{\mathrm{g}}(y)\right]^{\frac{1}{p(z, y)-1}} .
$$

As a result of the Minkowski inequality (see Theorem 2.13 in [15]) and inequality (27) in [2], we obtain that

$$
\begin{aligned}
& \left\|\frac{\mathrm{u}+v}{2}\right\|_{W_{0}^{s, p(z, y)}(\mathcal{M})}^{p^{\prime}(z, y)}+\left\|\frac{\mathrm{u}-v}{2}\right\|_{W_{0}^{s, p(z, y)}(\mathcal{M})}^{p^{\prime}(z, y)} \\
& \leq\left\{\int _ { \mathcal { M } \times \mathcal { M } } \left[\left(\left|\frac{(\mathrm{u}(z)-u(y))+(v(z)-v(y))}{2\left(d_{\mathrm{g}}(z, y)\right)^{\frac{n}{p(z, y)}}+s}\right|\right)^{p^{\prime}(z, y)}\right.\right. \\
& \left.\left.+\left(\left|\frac{(\mathrm{u}(z)-\mathrm{u}(y))-(v(z)-v(y))}{2\left(d_{\mathrm{g}}(z, y)\right)^{\frac{n}{p(z, y)}}+s}\right|\right)^{p^{\prime}(z, y)}\right]^{p(z, y)-1} d v_{\mathrm{g}}(z) d v_{\mathrm{g}}(y)\right\}^{\frac{1}{p(z, y)-1}} \\
& \leq\left(\frac{1}{2}\|\mathrm{u}\|_{W_{0}^{s, p(z, y)}(\mathcal{M})}^{p(z, y)}+\frac{1}{2}\|v\|_{\left.W_{0}^{s, p(z, y)}(\mathcal{M})\right)}^{p(z, y)}\right)^{p^{\prime}(z, y)-1}=1 .
\end{aligned}
$$

Hence,

$$
\left\|\frac{\mathrm{u}+v}{2}\right\|_{W_{0}^{s, p(z, y)}(M)}^{p^{\prime}(z, y)} \leq 1-\frac{\varepsilon^{p^{\prime}(z, y)}}{2^{p^{\prime}(z, y)}} .
$$

Taking $\delta=\delta(\varepsilon)$ such that $1-(\varepsilon / 2)^{p^{\prime}(z, y)}=(1-\delta)^{p^{\prime}(z, y)}$, from the Milman-Pettis theorem we obtain that $W_{0}^{s, p(z, y)}(\mathcal{M})$ is reflexive.

Now, we show that $W_{0}^{s, p(z, y)}(\mathcal{M})$ is a separable space. Define the operator

$$
\begin{aligned}
& T: W^{s, p(z, y)}(\mathcal{M}) \rightarrow L^{\hat{p}(z)}(\mathcal{M}) \times L^{p(z, y)}(\mathcal{M} \times \mathcal{M}), \\
& \mathrm{u} \mapsto T(\mathrm{u})=\left(\mathrm{u}(z), \frac{\mathrm{u}(z)-\mathrm{u}(y)}{d_{g}(z, y)^{\frac{n}{p(z, y)}+s}}\right) .
\end{aligned}
$$

Then

- $T$ is well defined.

- $T$ is an isometry. 
Indeed, for $\mathrm{u} \in W_{0}^{s, p(z, y)}(\mathcal{M})$, we obtain

$$
\begin{aligned}
\|T(\mathrm{u})\|_{L^{\hat{p}(z)(\mathcal{M}) \times L^{p(z, y)}(\mathcal{M} \times \mathcal{M})}} & =\|\mathrm{u}\|_{L^{\hat{p}(z)}(\mathcal{M})}+\left\|\frac{\mathrm{u}(z)-\mathrm{u}(y)}{d_{\mathrm{g}}(z, y)^{\frac{n}{p(z, y)}+s}}\right\|_{L^{p(z, y)}(\mathcal{M} \times \mathcal{M})} \\
& =\|\mathrm{u}\|_{W_{0}^{s, p(z, y)}(\mathcal{M})^{.}}
\end{aligned}
$$

So, $T\left(W_{0}^{s, p(z, y)}(\mathcal{M})\right)$ is a closed subspace of $L^{\hat{p}(z)}(\mathcal{M}) \times L^{p(z, y)}(\mathcal{M} \times \mathcal{M})$. Thanks to Proposition 3.17 in [15], we get that $T\left(W_{0}^{s, p(z, y)}(\mathcal{M})\right)$ is separable, therefore $W_{0}^{s, p(z, y)}(\mathcal{M})$ is also separable.

Lemma 3.4 Suppose that $(\mathcal{M}, \mathrm{g})$ satisfies property $B_{v o l}(\lambda, v)$ with finite volume, and (2) holds. Then $C_{0}^{\infty}(\mathcal{M})$ is dense in $W^{s, p(z, y)}(\mathcal{M})$.

Proof Consider the following real-valued function:

$$
f(t)= \begin{cases}1 & \text { if } t \leq 0 \\ 1-t & \text { if } 0 \leq t \leq 1 \\ 0 & \text { if } t \geq 1\end{cases}
$$

Let $\varphi \in C^{\infty}(\mathcal{M}) \cap W^{s, p(z, y)}(\mathcal{M})$, and let $y$ be a fixed point of $\mathcal{M}$ such that $\varphi_{v}(\alpha)=$ $\left.\varphi(\alpha) f\left(d_{\mathrm{g}}(y, \alpha)\right)\right)$, where $d_{\mathrm{g}}$ is the Riemannian distance associated to $\mathrm{g}$ and $v \in \mathbb{N}$. We can easily see that $\varphi_{v}(\alpha) \in W^{s, p(z, y)}(\mathcal{M})$ for $v \in \mathbb{N}$. Then, since $\mathcal{M}$ is a compact Riemannian $n$-manifolds, it can be covered by a finite number of charts $\left(\mathcal{Q}_{k}, \phi_{k}\right)_{k=1, \ldots, m}$. Let $\eta_{k}$ be a smooth partition of unity subordinate to the covering $\mathcal{Q}_{k}$. We can see that $h=\eta_{k} \varphi_{\nu} \circ \phi_{k}^{-1} \in$ $W^{s, p(z, y)}\left(\phi_{k}\left(\mathcal{Q}_{k}\right)\right)$.

So, by Lemma 3.2 in [7], we can extract a subsequence $h_{t} \in C^{\infty}\left(\mathbb{R}^{\ltimes}\right)$ such that $h_{t} \rightarrow$ $h$ strongly in $W^{s, p(z, y)}\left(\phi_{k}\left(\mathcal{Q}_{k}\right)\right)$ as $t \rightarrow \infty$. Thus, $h_{t} \circ \phi_{k} \in C^{\infty}(\mathcal{M})$ and $h_{t} \circ \phi_{k}$ converge strongly to $\eta_{k} \varphi_{v}$ in $W^{s, p(z, y)}(\mathcal{M})$ as $t \rightarrow \infty$.

Remark 3.2 We can also prove the previous lemma, without assuming condition (2), by using the following method:

For $\mathrm{u} \in C_{0}^{\infty}(\mathcal{M})$, we need to prove that

$$
\int_{\mathcal{M} \times \mathcal{M}} \frac{|\mathrm{u}(z)-\mathrm{u}(y)|^{p(z, y)}}{\left(d_{\mathrm{g}}(z, y)\right)^{n+s p(z, y)}} d v_{\mathrm{g}}(z) d v_{\mathrm{g}}(y)<\infty
$$

Notice that $\forall(z, y) \in \mathcal{M} \times \mathcal{M}$, we have

$$
|\mathrm{u}(z)-\mathrm{u}(y)| \leq\|D \mathrm{u}\|_{L^{\infty}(\mathcal{M})} d_{\mathrm{g}}(z, y), \quad|\mathrm{u}(z)-\mathrm{u}(y)| \leq 2\|\mathrm{u}\|_{L^{\infty}(\mathcal{M})} .
$$

Thus,

$$
|\mathrm{u}(z)-\mathrm{u}(y)|^{p(z, y)} \leq\|D \mathrm{u}\|_{L^{\infty}(\mathcal{M})}^{p(z, y)}\left(d_{\mathrm{g}}(z, y)\right)^{p(z, y)}, \quad \text { for all }(z, y) \in \mathcal{M} \times \mathcal{M},
$$

and

$$
|\mathrm{u}(z)-\mathrm{u}(y)|^{p(z, y)} \leq 2^{p(z, y)}\|\mathrm{u}\|_{L^{\infty}(\mathcal{M})}^{p(z, y)}, \quad \text { for all }(z, y) \in \mathcal{M} \times \mathcal{M}
$$


Hence,

$$
\begin{aligned}
& |\mathrm{u}(z)-\mathrm{u}(y)|^{p(z, y)} \leq\left(\|D \mathrm{u}\|_{L^{\infty}(\mathcal{M})}^{p^{+}}+\|D \mathrm{u}\|_{L^{\infty}(\mathcal{M})}^{p^{-}}\right)\left(d_{\mathrm{g}}(z, y)\right)^{p(z, y)}, \\
& \quad \text { for all }(z, y) \in \mathcal{M} \times \mathcal{M},
\end{aligned}
$$

and

$$
|\mathrm{u}(z)-\mathrm{u}(y)|^{p(z, y)} \leq 2^{p^{+}}\left(\|\mathrm{u}\|_{L^{\infty}(\mathcal{M})}^{p^{+}}+\|\mathrm{u}\|_{L^{\infty}(\mathcal{M})}^{p^{-}}\right), \quad \text { for all }(z, y) \in \mathcal{M} \times \mathcal{M} \text {. }
$$

Hence

$$
|\mathrm{u}(z)-\mathrm{u}(y)|^{p(z, y)} \leq 2^{p^{+}-1}\left(\|\mathrm{u}\|_{C^{1}(\mathcal{M})}^{p^{+}}+\|\mathrm{u}\|_{C^{1}(\mathcal{M})}^{p^{-}}\right) \min \left\{1,\left(d_{\mathrm{g}}(z, y)\right)^{p(z, y)}\right\} .
$$

Therefore, according to [30], we obtain

$$
\begin{aligned}
& \int_{\mathcal{M} \times \mathcal{M}} \frac{\left|\left(\eta_{s} \mathrm{u}\right)(z)-\left(\eta_{s} \mathrm{u}\right)(y)\right|^{p(z, y)}}{\left(d_{\mathrm{g}}(z, y)\right)^{n+s p(z, y)}} d v_{\mathrm{g}}(z) d v_{\mathrm{g}}(y) \\
& \leq \operatorname{vol}(\mathcal{M}) 2^{p^{+}-1}\left(\|\mathrm{u}\|_{C^{1}(\mathcal{M})}^{p^{+}}+\|\mathrm{u}\|_{C^{1}(\mathcal{M})}^{p^{-}}\right) \\
& \quad \times \int_{\mathcal{M} \times \mathcal{M}} \frac{\min \left\{1,\left(d_{\mathrm{g}}(z, y)\right)^{p(z, y)}\right\}}{\left(d_{\mathrm{g}}(z, y)\right)^{n+s p(z, y)}} d v_{\mathrm{g}}(z) d v_{\mathrm{g}}(y)<\infty
\end{aligned}
$$

where $\left(\eta_{s}\right)$ is a smooth partition of unity subordinate of the covering $B_{z_{k}}(r)$ for any $k$, and $B_{z_{k}}(r)$ denotes the Euclidean ball of $\mathbb{R}^{n}$ with center $z_{k}$ and radius $r$. Then we deduce that, for $\mathrm{u} \in C_{0}^{\infty}(\mathcal{M})$,

$$
\int_{\mathcal{M} \times \mathcal{M}} \frac{|\mathrm{u}(z)-\mathrm{u}(y)|^{p(z, y)}}{\left(d_{\mathrm{g}}(z, y)\right)^{n+s p(z, y)}} d \nu_{\mathrm{g}}(z) d v_{\mathrm{g}}(y)<\infty
$$

Thus $\mathrm{u} \in W^{s, p(z, y)}(\mathcal{M})$.

Now, we will extend an embedding result between $W^{1, p(z, y)}(\mathcal{M})$ and $W^{s, p(z, y)}(\mathcal{M})$ to manifolds.

Lemma 3.5 Suppose that the smooth complete compact Riemannian n-manifold $(\mathcal{M}, \mathrm{g})$ has property $B_{v o l}(\lambda, v)$ for some $(\lambda, v), p \in C(\mathcal{M} \times \mathcal{M},(1,+\infty))$, and $s \in(0,1)$. Then $\|\mathrm{u}\|_{W^{s, p(z, y)}(\mathcal{M})} \leq C\|\mathrm{u}\|_{W^{1, p(z, y)}(\mathcal{M})}$, where $C=C\left(n, s, \lambda, v, p^{+}, p^{-}\right)$. In particular, $W^{1, p(z, y)}(\mathcal{M}) \sqsubseteq$ $W^{s, p(z, y)}(\mathcal{M})$.

Proof For the sake of convenience, let $[u]_{W^{1, p(z, y)}(\mathcal{M})}=1$ and set

$$
C=\sup _{(z, y) \in \mathcal{M} \times \mathcal{M}}\left(d_{g}(z, y)\right)^{(1-s) p(z, y)} .
$$

Then

$$
\int_{\mathcal{M} \times \mathcal{M}} \frac{|\mathrm{u}(z)-\mathrm{u}(y)|^{p(z, y)}}{C\left(d_{g}(z, y)\right)^{n+s p(z, y)}} d v_{g}(z) d v_{g}(y)
$$




$$
\begin{aligned}
& =\int_{\mathcal{M} \times \mathcal{M}} \frac{|\mathrm{u}(z)-\mathrm{u}(y)|^{p(z, y)}}{\left(d_{g}(z, y)\right)^{n+p(z, y)}} \frac{\left(d_{g}(z, y)\right)^{(1-s) p(z, y)}}{C} d v_{g}(z) d v_{g}(y) \\
& \leq \int_{\mathcal{M} \times \mathcal{M}} \frac{|\mathrm{u}(z)-\mathrm{u}(y)|^{p(z, y)}}{\left(d_{g}(z, y)\right)^{n+p(z, y)}} d v_{g}(z) d v_{g}(y) \\
& \leq 1 .
\end{aligned}
$$

Thus, $[u]_{W^{s, p}(z, y)(\mathcal{M})} \leq C[u]_{W^{1, p(z, y)}(\mathcal{M})}$.

Hence,

$$
\|u\|_{W^{s, p(z, y)}(\mathcal{M})} \leq C\|u\|_{W^{1, p(z, y)}(\mathcal{M})} .
$$

Remark 3.3 We can also prove the previous lemma using the same technique as that of [29, Lemma 2.6].

Theorem 3.1 Let $\mathcal{M}$ be a compact Riemannian manifold, $p \in C(\mathcal{M} \times \mathcal{M},(1 ; \infty)), s \in(0,1)$ with $\operatorname{sp}(z, y)<n$ and $q \in C(\mathcal{M},(1 ; \infty))$. Assume that

$$
1<q^{-}=\min _{z \in \mathcal{M}} q(z) \leq q(z)<\frac{n \hat{p}(z)}{n-s \hat{p}(z)} \quad \text { for all } z \in \overline{\mathcal{M}},
$$

then $W^{s, p(z, y)}(\mathcal{M}) \hookrightarrow L^{q(z)}(\mathcal{M})$ is a continuous and compact embedding.

Proof The demonstration of this theorem is based on an idea introduced in $[1,21,28,29]$. Let $\varphi: V \subset \mathcal{M} \rightarrow \mathbb{R}^{n}$ be an arbitrary local chart on $\mathcal{M}$, and $G \subset \mathcal{M}$ an open set with compact closure and contained in $V$. Take $\left\{G_{l}\right\}_{l=1, \ldots, k}$ to be a finite subcovering of $\mathcal{M}$ such that $G_{l}$ is homeomorphic to the open unit ball $B_{0}(1)$ of $\mathbb{R}^{n}$ and, for any $l$, the components $\mathrm{g}_{i j}^{l}$ of $\mathrm{g}$ in $\left(\mathrm{g}_{l}, V_{l}\right)$ satisfy

$$
\frac{1}{\alpha \delta_{i j}}<\mathrm{g}_{i j}^{l}<\alpha \delta_{i j}
$$

as bilinear forms, for some constant $\alpha>1$. Let $\left\{\pi_{l}\right\}_{l=1, \ldots, k}$ be a smooth partition of unity subordinate to the finite covering $\left\{G_{l}\right\}_{l=1, \ldots, k}$. It is clear that if $\mathrm{u} \in W^{s, p(z, y)}(\mathcal{M})$, then $\pi_{l} \mathrm{u} \in W^{s, p(z, y)}\left(G_{l}\right)$ and $\left(\varphi_{l}^{-1}\right) *\left(\pi_{l} \mathrm{u}\right) \in W^{s, p\left(\varphi_{l}^{-1}(z, y)\right)}\left(B_{0}(1)\right)$ with $\mathrm{u}=\sum_{l=1}^{k} \pi_{l} \mathrm{u}$. According to Lemma 3.5, the Sobolev embedding theorem [1, 21, 29], we get the continuous and compact embedding

$$
W^{s, p(z, z)}\left(G_{l}\right) \hookrightarrow L^{q(z)}\left(G_{l}\right) \quad \text { for any } l=1, \ldots, k
$$

Thus, we can conclude that $W^{s, p(z, y)}(\mathcal{M}) \subset L^{q(z)}(\mathcal{M})$, and the embedding is continuous and compact.

\section{Application}

In this part, as an application, we give an existence result to the following problem:

$$
(\mathcal{P}) \quad\left\{\begin{array}{l}
\left(-\Delta_{g}\right)_{p(z,)}^{s} \mathrm{u}(z)+\mathcal{V}(z)|\mathrm{u}(z)|^{q(z)-2} \mathrm{u}=h(z, \mathrm{u}(z)) \quad \text { in } \mathcal{Q} \\
\left.\mathrm{u}\right|_{\partial \mathcal{Q}}=0
\end{array}\right.
$$


where $s \in(0,1), p \in C(\mathcal{M} \times \mathcal{M},(1 ; \infty))$ with $s p(z, y)<n, q: \overline{\mathcal{Q}} \rightarrow(1, \infty)$ satisfy the following condition:

$$
1<q^{-} \leq q^{+}<p^{-} \leq p^{+}<\frac{n \hat{p}(z)}{n-s \hat{p}(z)}
$$

$(\mathcal{M}, \mathrm{g})$ is a smooth complete compact Riemannian $n$-manifold, $\mathcal{Q} \subset \mathcal{M}$ is an open bounded set with smooth boundary $\partial \mathcal{Q}, h: \mathcal{Q} \times \mathbb{R} \rightarrow \mathbb{R}$ is a Carathéodory function satisfying the following assumptions:

$\left(h_{1}\right)$ (AR-condition) There exist $\beta>p^{+}$and some $I>0$ such that, for each $|\alpha|>I$, we have

$$
0<\int_{\mathcal{M}} H(z, \alpha) d v_{\mathrm{g}}(z) \leq \int_{\mathcal{M}} h(z, \alpha) \frac{\alpha}{\beta} d v_{\mathrm{g}}(z) \quad \text { a.e. } z \in \mathcal{M},
$$

where $H(z, \alpha)=\int_{0}^{\alpha} h(z, v) d v$ is the primitive of $h(z, \alpha)$,

$\left(h_{2}\right) h(z, 0)=0$,

$\left(h_{3}\right) \lim _{|\alpha| \rightarrow 0} \frac{h(z, \alpha)}{|\alpha|^{q(z)-1}}=0$ uniformly a.e. $z \in \mathcal{M}$,

and $\left(-\Delta_{\mathrm{g}}\right)_{p(z,)}^{s} \mathrm{u}(z)$ is the fractional $p(z, \cdot)$-Laplace operator which (up to normalization factors) may be defined as

$$
\left(-\Delta_{\mathrm{g}}\right)_{p(z, \dot{)})}^{s} \mathrm{u}(z)=2 \lim _{\epsilon \rightarrow 0^{+}} \int_{M \backslash B_{\epsilon}(z)} \frac{|\mathrm{u}(z)-\mathrm{u}(y)|^{p(z, y)-2}(\mathrm{u}(z)-\mathrm{u}(y))}{\left(d_{\mathrm{g}}(z, y)\right)^{n+s p(z, y)}} d v_{\mathrm{g}}(y),
$$

for $z \in \mathcal{M}$, where $B_{\epsilon}(z)$ denotes the geodesic ball of $\mathcal{M}$ with center $z$ and radius $\epsilon$ and $d_{\mathrm{g}}(z, y)$ defines a distance on $\mathcal{M}$ whose topology coincides with the original one. The vanishing potential satisfies the following assumptions:

(i) $\mathcal{V}: \mathcal{M} \rightarrow \mathbb{R}$ is a continuous function, and there exist $\theta>0, \gamma>0$ such that

$\mathcal{V}(z)>\theta>0$ for all $z \in \mathcal{M}$, and

$$
\int_{\mathcal{M}} \mathcal{V}(z)|\mathrm{u}(z)|^{q(z)} d v_{\mathrm{g}}(z) \leq \gamma\|\mathrm{u}\|_{W^{s, p(z, y)}(\mathcal{M})^{\prime}}^{q(z)}
$$

for all $\mathrm{u} \in W_{0}^{s, p(z, y)}(\mathcal{M})$.

(ii) $\mathcal{V}(z) \rightarrow+\infty$ as $|z| \rightarrow+\infty$.

Definition 4.1 A measurable function $\mathrm{u} \in W_{0}^{s, p(z, y)}(\mathcal{M})$ is said to be a weak solution of $(\mathcal{P})$ if

$$
\begin{aligned}
& \int_{M \times M} \frac{|\mathrm{u}(z)-\mathrm{u}(y)|^{p(z, y)-2}(\mathrm{u}(z)-\mathrm{u}(y))(\varphi(z)-\varphi(y))}{\left(d_{g}(z, y)\right)^{n+s p(z)}} d v_{g}(z) d v_{g}(y) \\
& \quad+\int_{M} \mathcal{V}(z)|\mathrm{u}(z)|^{q(z)-2} u(x) \varphi(z) d v_{g}(z) \\
& =\int_{M} h(z, \mathrm{u}(z)) \varphi(z) d v_{g}(z), \quad \text { for all } \varphi \in W_{0}^{s, p(z, y)}(\mathcal{M}) .
\end{aligned}
$$

Theorem 4.1 Under assumptions $\left(h_{1}\right)-\left(h_{3}\right),(4)$ and $(i)-(i i)$, if $(M, g)$ satisfies the property $B_{\text {vol }}(\lambda, v)$, then problem $(\mathcal{P})$ possesses at least one weak solution. 
Proof Consider the functional $E: W_{0}^{s, p(z, y)}(\mathcal{M}) \rightarrow \mathbb{R}$ defined by

$$
E(\mathrm{u})=A(\mathrm{u})-B(\mathrm{u})
$$

where

$$
A(\mathrm{u})=\int_{\mathcal{M} \times \mathcal{M}} \frac{1}{p(z, y)} \frac{|\mathrm{u}(z)-\mathrm{u}(y)|^{p(z, y)}}{\left(d_{\mathrm{g}}(z, y)\right)^{n+s p(z, y)}} d v_{\mathrm{g}}(z) d v_{\mathrm{g}}(y)+\int_{\mathcal{M}} \frac{\mathcal{V}(z)}{q(z)}|\mathrm{u}(z)|^{q(z)} d v_{\mathrm{g}}(z)
$$

and

$$
B(\mathrm{u})=\int_{\mathcal{M}} H(z, \mathrm{u}(z)) d v_{\mathrm{g}}(z)
$$

Lemma 4.1 Assume that the assumptions (i)-(iii) hold. Then $A \in C^{1}\left(W_{0}^{s, p(z, y)}(\mathcal{M})\right)$ and

$$
\begin{aligned}
\left\langle A^{\prime}(\mathrm{u}), \varphi\right\rangle= & \int_{\mathcal{M} \times \mathcal{M}} \frac{|\mathrm{u}(z)-\mathrm{u}(y)|^{p(z, y)-2}(\mathrm{u}(z)-\mathrm{u}(y))(\varphi(z)-\varphi(y))}{\left(d_{\mathrm{g}}(z, y)\right)^{n+s p(z, y)}} d \nu_{\mathrm{g}}(z) d \nu_{\mathrm{g}}(y) \\
& +\int_{\mathcal{M}} \mathcal{V}(z)|\mathrm{u}(z)|^{q(z)-2} \mathrm{u}(z) \varphi(z) d \nu_{\mathrm{g}}(z),
\end{aligned}
$$

for all $\mathrm{u}, \varphi \in W_{0}^{s, p(z, y)}(\mathcal{M})$.

Proof For $\mathrm{u} \in W_{0}^{s, p(z, y)}(\mathcal{M})$, we have

$$
\begin{aligned}
& A(\mathrm{u})=\int_{\mathcal{M} \times \mathcal{M}} \frac{1}{p(z, y)} \cdot \frac{|\mathrm{u}(z)-\mathrm{u}(y)|^{p(z, y)}}{\left(d_{\mathrm{g}}(z, y)\right)^{n+s p(z, y)}} d v_{\mathrm{g}}(z) d v_{\mathrm{g}}(y)+\int_{\mathcal{M}} \frac{\mathcal{V}(z)}{q(z)}|\mathrm{u}(z)|^{q(z)} d v_{\mathrm{g}}(z) \\
& \leq \frac{1}{p^{-}} \int_{\mathcal{M} \times \mathcal{M}} \frac{|\mathrm{u}(z)-\mathrm{u}(y)|^{p(z, y)}}{\left(d_{\mathrm{g}}(z, y)\right)^{n+s p(z, y)}} d v_{\mathrm{g}}(z) d \nu_{\mathrm{g}}(y)+\frac{1}{q^{-}} \int_{\mathcal{M}} \mathcal{V}(z)|\mathrm{u}(z)|^{q(z)} d v_{\mathrm{g}}(z) \\
& \leq \frac{1}{p^{-}}\left(\|\mathrm{u}\|_{W_{0}^{s, p(z, y)}(\mathcal{M})}^{p^{+}}+\|\mathrm{u}\|_{W_{0}^{s, p(z, y)}(\mathcal{M})}^{p^{-}}\right)+\frac{\gamma}{q^{+}}\left(\|\mathrm{u}\|_{W_{0}^{s, p(z, y)}(\mathcal{M})}^{q^{+}}+\|\mathrm{u}\|_{W_{0}^{s, p(z, y)}(\mathcal{M})}^{q^{-}}\right) \\
& \text {(using Lemma } 3.1 \text { and (i)) } \\
& <+\infty \text {. }
\end{aligned}
$$

Hence, $A$ is well defined.

To prove that $A \in C^{1}\left(W_{0}^{s, p(z, y)}(\mathcal{M})\right)$, we consider $\left\{\mathrm{u}_{t}\right\} \subset W_{0}^{s, p(z, y)}(\mathcal{M})$ such that $\mathrm{u}_{t} \rightarrow \mathrm{u}$ strongly in $W_{0}^{s, p(z, y)}(\mathcal{M})$ as $t \rightarrow+\infty$. Then, we have

$$
\lim _{t \rightarrow+\infty} \int_{\mathcal{M} \times \mathcal{M}}\left[\frac{\left|\mathrm{u}_{t}(z)-\mathrm{u}_{t}(y)\right|^{p(z, y)}}{\left(d_{\mathrm{g}}(z, y)^{n+s p(z, y)}\right.}-\frac{|\mathrm{u}(z)-\mathrm{u}(y)|^{p(z, y)}}{\left(d_{\mathrm{g}}(z, y)^{n+s p(z, y)}\right.}\right] d v_{\mathrm{g}}(z) d v_{\mathrm{g}}(y)=0 .
$$

Without losing generality, we further assume that $\mathrm{u}_{t} \rightarrow \mathrm{u}$ a.e. in $\mathcal{M}$ as $t \rightarrow+\infty$. Using (6), we get that

$$
\left\{\frac{\left|\mathrm{u}_{t}(z)-\mathrm{u}_{t}(y)\right|^{p(z, y)}\left(\mathrm{u}_{t}(z)-\mathrm{u}_{t}(y)\right)}{\left(d_{\mathrm{g}}(z, y)\right)^{n+s p(z, y)}}\right\}_{t}
$$


is bounded in $L^{p(z, y)}(\mathcal{M} \times \mathcal{M})$ and, according to Brezis-Lieb lemma in [23], we obtain

$$
\lim _{t \rightarrow+\infty} \int_{\mathcal{M} \times \mathcal{M}}\left(\frac{\left|\mathrm{u}_{t}(z)-\mathrm{u}_{t}(y)\right|^{p(z, y)}}{\left(d_{\mathrm{g}}(z, y)\right)^{n+s p(z, y)}}-\frac{|\mathrm{u}(z)-\mathrm{u}(y)|^{p(z, y)}}{\left(d_{\mathrm{g}}(z, y)\right)^{n+s p(z, y)}}\right) d v_{\mathrm{g}}(z) d v_{\mathrm{g}}(y)=0
$$

Similarly,

$$
\left.\lim _{t \rightarrow+\infty} \int_{\mathcal{M}} \mathcal{V}(z)|| \mathrm{u}_{t}(z)\right|^{q(z)-2} \mathrm{u}_{t}(z)-\left.|\mathrm{u}(z)|^{q(z)-2} \mathrm{u}(z)\right|^{q(z)} d v_{\mathrm{g}}(z)=0
$$

Hölder's inequality now gives

$$
\begin{aligned}
& \left\|A^{\prime}\left(\mathrm{u}_{t}\right)-A^{\prime}(\mathrm{u})\right\|_{\left(W_{0}^{s, p(z, y)}(\mathcal{M})\right)^{\prime}} \\
& \quad=\sup _{\varphi \in W_{0}^{s, p(z, y)}(\mathcal{M}) ;\|\varphi\|_{W_{0}^{s, p(z, y)}(\mathcal{M})}=1} \mid\left\langle A^{\prime}\left(\mathrm{u}_{t}\right)-A^{\prime}(\mathrm{u}), \varphi\right\rangle \rightarrow 0 \quad \text { as } t \rightarrow+\infty .
\end{aligned}
$$

Hence, $A \in C^{1}\left(W_{0}^{s, p(z, y)}(\mathcal{M})\right)$. Finally, we can easily verify that the functional $A$ is Gâteaux differentiable on $W_{0}^{s, p(z, y)}(\mathcal{M})$ and (5) holds for all $u, \varphi \in W_{0}^{s, p(z, y)}(\mathcal{M})$.

Lemma 4.2 The functional E satisfies the mountain pass geometry in the sense that

(i) $E(0)=0$.

(ii) There exist $\zeta, \mu>0$ such that $E(\mathrm{u}) \geq \mu$ if $\|\mathrm{u}\|_{W_{0}^{s, p(z, y)}(\mathcal{M})}>\zeta$.

(iii) There exists $\mathrm{u}$, with $\|\mathrm{u}\|_{W_{0}^{s, p(z, y)}(\mathcal{M})}>\zeta$, such that $E(\mathrm{u}) \leq 0$.

\section{Proof}

(i) $E(0)=0$ is obvious.

(ii) According to $\left(h_{1}\right),(i)$, Proposition 2.4, Lemma 3.1, and Theorem 3.1, we have

$$
\begin{aligned}
E(\mathrm{u})= & \int_{\mathcal{M} \times \mathcal{M}} \frac{1}{p(z, y)} \frac{|\mathrm{u}(z)-\mathrm{u}(y)|^{p(z, y)}}{\left(d_{\mathrm{g}}(z, y)\right)^{n+s p(z, y)}} d v_{\mathrm{g}}(z) d v_{\mathrm{g}}(y) \\
& +\int_{\mathcal{M}} \frac{\mathcal{V}(z)}{q(z)}|\mathrm{u}(z)|^{q(z)} d v_{\mathrm{g}}(z)-\int_{\mathcal{M}} H(z, \mathrm{u}(z)) d v_{\mathrm{g}}(z) \\
\geq & \frac{1}{p^{+}} \varrho_{p(\cdot, \cdot)}(\mathrm{u})+\frac{\theta}{q^{+}}\|\mathrm{u}\|_{L^{q(z)}(\mathcal{M})}^{q^{+}}-\frac{1}{\beta}\|\mathrm{u}\|_{W_{0}^{q^{s}, p(z, y)}(\mathcal{M})}^{q^{-}} \\
\geq & \frac{1}{p^{+}}\|\mathrm{u}\|_{W_{0}^{p^{+}, p(z, y)}(\mathcal{M})}+\frac{\theta C^{q^{+}}}{q^{+}}\|\mathrm{u}\|_{W_{0}^{q^{+}, p(z, y)}(\mathcal{M})}^{p^{(}}-\frac{1}{p^{+}}\|\mathrm{u}\|_{W_{0}^{s, p(z, y)}(\mathcal{M})}^{q^{-}}
\end{aligned}
$$

Since $p^{+}>q^{+} \geq q^{-}$, we deduce that $E(\mathrm{u})>0$.

(iii) According to Lemma 3.1, $\left(h_{1}\right),\left(h_{3}\right)$, and (i), we have for $t>0$ and $\mathbf{u} \neq 0$,

$$
\begin{aligned}
E(t \mathrm{u})= & \int_{\mathcal{M} \times \mathcal{M}} \frac{t^{p(z, y)}}{p(z, y)} \frac{|\mathrm{u}(z)-\mathrm{u}(y)|^{p(z, y)}}{\left(d_{\mathrm{g}}(z, y)\right)^{n+s p(z, y)}} d v_{\mathrm{g}}(z) d v_{\mathrm{g}}(y) \\
& +\int_{\mathcal{M}} \frac{\mathcal{V}(z)}{q(z)} t^{q(z)}|\mathrm{u}(z)|^{q(z)} d v_{\mathrm{g}}(z)-\int_{\mathcal{M}} H(z, t \mathrm{u}(z)) d v_{\mathrm{g}}(z) \\
\leq & \frac{t^{p^{+}}}{p^{-}} \varrho_{p(\cdot, \cdot)}(\mathrm{u})+\frac{\gamma t^{q^{-}}}{q^{-}}\|\mathrm{u}\|_{W_{0}^{s, p(z, y)}(\mathcal{M})}^{q^{-}}
\end{aligned}
$$




$$
\leq \frac{t^{p^{+}}}{p^{-}}\|\mathrm{u}\|_{W_{0}^{s, p(z, y)}(\mathcal{M})}^{p^{-}}+\frac{\gamma t^{q^{-}}}{q^{-}}\|\mathrm{u}\|_{W_{0}^{s, p(z, y)}(\mathcal{M})}^{q^{-}}
$$

Since $q^{-}<p^{-}$, for $t$ small enough, we infer that $E(t \mathrm{u})<0$.

This completes the demonstration of the lemma.

Lemma 4.3 Let $(\mathcal{M}, \mathrm{g})$ be a smooth complete compact Riemannian n-manifold and $p(\cdot) \in$ $C_{+}(\mathcal{M} \times \mathcal{M})$. Assume that assumptions $\left(h_{1}\right)-\left(h_{3}\right)$ and $(i)-(i i)$ hold. Then the functional $E$ satisfies the Palais-Smale condition.

Proof Let $\left(\mathrm{u}_{m}\right)_{m \in \mathbb{N}} \subset W_{0}^{s, p(z, y)}(\mathcal{M})$ be such that $J\left(\mathrm{u}_{m}\right)$ is bounded and $J^{\prime}\left(\mathrm{u}_{m}\right) \rightarrow 0$ as $m \rightarrow$ $\infty$. Then there exists $C>0$ such that $d J\left(\mathrm{u}_{m}, \mathrm{u}_{m}\right) \leq C\left\|\mathrm{u}_{m}\right\|_{W_{0}^{s, p(z, y)}}$ and $\left|J\left(\mathrm{u}_{m}\right)\right|<C$. We prove that $\left(\mathrm{u}_{m}\right)_{m \in \mathbb{N}}$ is bounded in $W_{0}^{s, p(z, y)}(\mathcal{M})$.

We prove the statement by the method of contradiction. Indeed, if the assertion were not true, up to a subsequence, $\left\|\mathrm{u}_{m}\right\|_{W_{0}^{s, p(z, y)}(\mathcal{M})} \rightarrow \infty$ as $m \rightarrow \infty$. From $\left(h_{1}\right)$, we have

$$
C+C\left\|\mathrm{u}_{m}\right\|_{W_{0}^{s, p(z, y)}(\mathcal{M})} \geq E\left(\mathrm{u}_{m}\right)-\frac{1}{\beta}\left\langle E^{\prime}\left(\mathrm{u}_{m}\right), \mathrm{u}_{m}\right\rangle \geq\left(\frac{1}{p^{+}}-\frac{1}{\beta}\right)\left\|\mathrm{u}_{m}\right\|_{W_{0}^{s, p(z, y)}(\mathcal{M})}^{p^{+}} .
$$

This is a contradiction as $\left\|\mathrm{u}_{m}\right\|_{W_{0}^{s, p(z, y)}(\mathcal{M})} \rightarrow \infty$. Since $W_{0}^{s, p(z, y)}(\mathcal{M})$ is a reflexive Banach space, there exists $\mathrm{u} \in W_{0}^{s, p(z, y)}(\mathcal{M})$ such that, up to a subsequence,

$$
\begin{aligned}
& \mathrm{u}_{m} \rightarrow \mathrm{u} \quad \text { weakly in } W_{0}^{s, p(z, y)}(\mathcal{M}), \\
& \mathrm{u}_{m} \rightarrow \mathrm{u} \quad \text { a.e. in } \mathcal{M}, \\
& \mathrm{u}_{m} \rightarrow \mathrm{u} \quad \text { strongly in } L^{\gamma(z)}(\mathcal{M}) \text { for } 1 \leq \gamma(z)<p_{s}^{*}(z) .
\end{aligned}
$$

Since $\mathrm{u}_{m} \longrightarrow \mathrm{u}$ a.e. in $\mathcal{M}$, we have that

$$
\frac{\left|\mathrm{u}_{m}(z)-\mathrm{u}_{m}(y)\right|^{p(z, y)-2}\left(\mathrm{u}_{m}(z)-\mathrm{u}_{m}(y)\right)}{\left(d_{\mathrm{g}}(z, y)\right)^{\left(\frac{n}{p(z, y)}+s\right)(p(z, y)-1)}} \rightarrow \frac{|\mathrm{u}(z)-\mathrm{u}(y)|^{p(z, y)-2}(\mathrm{u}(z)-\mathrm{u}(y))}{\left(d_{\mathrm{g}}(z, y)\right)^{\left(\frac{n}{p(z, y)}+s\right)(p(z, y)-1)}}
$$

a.e. $(z, y) \in \mathcal{M} \times \mathcal{M}$. On the other hand, $\left\|\mathrm{u}_{m}\right\|_{W_{0}^{s, p(z, y)}(\mathcal{M})}$ is uniformly bounded and this implies that there exists a constant $C>0$ such that

$$
\int_{\mathcal{M} \times \mathcal{M}}\left(\frac{\left|\mathrm{u}_{m}(z)-\mathrm{u}_{m}(y)\right|}{d_{\mathrm{g}}(z, y)^{\frac{n}{p(z, y)}+s}}\right)^{p(z, y)} d v_{\mathrm{g}}(z) d v_{\mathrm{g}}(y) \leq C \quad \text { for all } m \geq 1
$$

that is,

$$
\int_{\mathcal{M} \times \mathcal{M}}\left|\frac{\left|\mathbf{u}_{m}(z)-\mathbf{u}_{m}(y)\right|^{p(z, y)-2}\left(\mathbf{u}_{m}(z)-\mathrm{u}_{m}(y)\right)}{d_{\mathrm{g}}(z, y)^{\left(\frac{n}{p(z, y)}+s\right)(p(z, y)-1)}}\right|^{\frac{p(z, y)}{p(z, y)-1}} d v_{\mathrm{g}}(z) d v_{\mathrm{g}}(y) \leq C .
$$

Therefore,

$$
\frac{\left|\mathrm{u}_{m}(z)-\mathrm{u}_{m}(y)\right|^{p(z, y)-2}\left(\mathrm{u}_{m}(z)-\mathrm{u}_{m}(y)\right)}{d_{\mathrm{g}}(z, y)^{\left(\frac{n}{p(z, y)}+s\right)(p(z, y)-1)}}-\frac{|\mathrm{u}(z)-\mathrm{u}(y)|^{p(z, y)-2}(\mathrm{u}(z)-\mathrm{u}(y))}{d_{\mathrm{g}}(z, y)^{\left(\frac{n}{p(z, y)}+s\right)(p(z, y)-1)}}
$$


weakly in $L^{p^{\prime}(z, y)}(\mathcal{M} \times \mathcal{M})$ with $p^{\prime}(z, y)=\frac{p(z, y)}{p(z, y)-1}$. If $v \in W_{0}^{s, p(z, y)}(\mathcal{M})$, it follows that $\frac{v(z)-v(y)}{d_{\mathrm{g}}(z, y)^{\frac{\eta}{p(z, y)}}+s} \in L^{p(z, y)}(\mathcal{M} \times \mathcal{M})$. As a result,

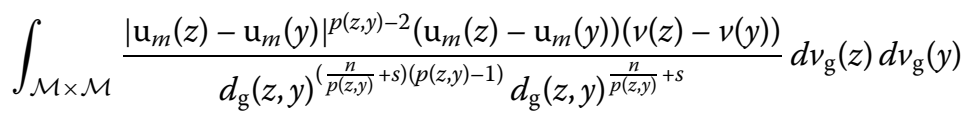

$$
\begin{aligned}
& \rightarrow \int_{\mathcal{M} \times \mathcal{M}} \frac{|\mathrm{u}(z)-\mathrm{u}(y)|^{p(z, y)-2}(\mathrm{u}(z)-\mathrm{u}(y))(v(z)-v(y))}{d_{\mathrm{g}}(z, y)^{n+s p(z, y)}} d v_{\mathrm{g}}(z) d v_{\mathrm{g}}(y) .
\end{aligned}
$$

By Vitali's theorem, we have $E^{\prime}(\mathrm{u})=0$. We define the sequence $w_{n}=\mathrm{u}_{m}-\mathrm{u}$. Since $\left(\mathrm{u}_{m}\right)_{m \in \mathbb{N}}$ is uniformly bounded and $\mathrm{u}_{m} \rightarrow \mathrm{u}$ a.e. in $\mathcal{M}$, thanks to Brezis-Lieb lemma in [23], we obtain that

$$
\begin{aligned}
& \int_{\mathcal{M} \times \mathcal{M}} \frac{\left|\mathrm{u}_{m}(z)-\mathrm{u}_{m}(y)\right|^{p(z, y)}}{d_{\mathrm{g}}(z, y)^{n+s p(z, y)}} d v_{\mathrm{g}}(z) d v_{\mathrm{g}}(y) \\
& =\int_{\mathcal{M} \times \mathcal{M}} \frac{\left|w_{m}(z)-w_{m}(y)\right|^{p(z, y)}}{d_{\mathrm{g}}(z, y)^{n+s p(z, y)}} d v_{\mathrm{g}}(z) d v_{\mathrm{g}}(y) \\
& \quad+\int_{\mathcal{M} \times \mathcal{M}} \frac{|\mathrm{u}(z)-\mathrm{u}(y)|^{p(z, y)}}{d_{\mathrm{g}}(z, y)^{n+s p(z, y)}} d v_{\mathrm{g}}(z) d v_{\mathrm{g}}(y)+o(1) .
\end{aligned}
$$

Therefore, a straightforward computation yields

$$
\begin{aligned}
\lim _{m \rightarrow \infty} E\left(\mathrm{u}_{m}\right)= & \lim _{m \rightarrow \infty} \int_{\mathcal{M} \times \mathcal{M}} \frac{\left|w_{m}(z)-w_{m}(y)\right|^{p(z, y)}}{d_{\mathrm{g}}(z, y)^{n+s p(z, y)}} d v_{\mathrm{g}}(z) d v_{\mathrm{g}}(y) \\
& -\int_{\mathcal{M}} H(z, \mathrm{u}(z)) d v_{\mathrm{g}}(z)+\int_{\mathcal{M}} \frac{\mathcal{V}(z)}{q(z)}|\mathrm{u}(z)|^{q(z)} d v_{\mathrm{g}}(z) \\
& +\int_{\mathcal{M} \times \mathcal{M}} \frac{1}{p(z, y)}|\mathrm{u}(z)-\mathrm{u}(y)|^{p(z, y)} d_{\mathrm{g}}(z, y)^{n+s p(z, y)} d v_{\mathrm{g}}(z) d v_{\mathrm{g}}(y) .
\end{aligned}
$$

Using $E^{\prime}\left(\mathrm{u}_{m}\right) \rightarrow 0$ as $m \rightarrow \infty$, we have

$$
\begin{aligned}
& \int_{\mathcal{M} \times \mathcal{M}} \frac{1}{p(z, y)} \frac{\left|w_{m}(z)-w_{m}(y)\right|^{p(z, y)}}{d_{\mathrm{g}}(z, y)^{n+s p(z, y)}} d v_{\mathrm{g}}(z) d v_{\mathrm{g}}(y) \\
& \rightarrow \int_{\mathcal{M}} \mathcal{V}(z)|\mathrm{u}(z)|^{q(z)} d v_{\mathrm{g}}(z)-\int_{\mathcal{M}} h(z, \mathrm{u}(z)) \mathrm{u}(z) d v_{\mathrm{g}}(z) \\
& \quad+\int_{\mathcal{M} \times \mathcal{M}} \frac{1}{p(z, y)} \frac{\left|\mathrm{u}_{m}(z)-\mathrm{u}_{m}(y)\right|^{p(z, y)}}{d_{\mathrm{g}}(z, y)^{n+s p(z, y)}} d v_{\mathrm{g}}(z) d v_{\mathrm{g}}(y) .
\end{aligned}
$$

As $E^{\prime}(\mathrm{u})=0$, we have

$$
\lim _{m \rightarrow \infty} \int_{\mathcal{M} \times \mathcal{M}} \frac{1}{p(z, y)} \frac{\left|w_{m}(z)-w_{m}(y)\right|^{p(z, y)}}{d_{\mathrm{g}}(z, y)^{n+s p(z, y)}} d v_{\mathrm{g}}(z) d v_{\mathrm{g}}(y)=0
$$

So, finally, from Lemmas 4.2 and 4.3 , we conclude that our problem $(\mathcal{P})$ possesses a weak solution. 


\section{Acknowledgements}

The authors would like to thank the referees for their suggestions and helpful comments which have improved the presentation of the original manuscript.

Funding

Repovš was supported by the Slovenian Research Agency grants P1-0292, N1-0114, and N1-0083.

\section{Availability of data and materials}

Not applicable.

\section{Declarations}

\section{Competing interests}

The authors declare that they have no competing interests.

\section{Authors' contributions}

The authors declare that their contributions are equal. All authors read and approved the final manuscript.

\section{Author details}

${ }^{1}$ National School of Applied Sciences, Sidi Mohamed Ben Abdellah University, Fez, Morocco. ${ }^{2}$ Faculty of Sciences Dhar El Mahraz, Sidi Mohamed Ben Abdellah University, Fez, Morocco. ${ }^{3}$ Faculty of Mathematics and Physics, University of Ljubljana, Jadranska 19, SI-1000, Ljubljana, Slovenia.

\section{Publisher's Note}

Springer Nature remains neutral with regard to jurisdictional claims in published maps and institutional affiliations.

Received: 15 November 2021 Accepted: 1 February 2022 Published online: 09 February 2022

\section{References}

1. Aberqi, A., Bennouna, J., Benslimane, O., Ragusa, M.A.: Existence results for double phase problem in Sobolev-Orlicz spaces with variable exponents in complete manifold. Mediterr. J. Math. (2022, to appear). arXiv:2110.03289 [math.AP]

2. Adams, R., Fournier, J.F.: Sobolev Spaces. Academic Press, New York (1975)

3. Aubin, T.: Nonlinear Analysis on Manifolds. Monge-Ampere Equations, vol. 252. Springer, Berlin (1982)

4. Ayazoglu, R., Saraç, Y., Şener, S., Alisoy, G.: Existence and multiplicity of solutions for a Schrödinger-Kirchhoff type equation involving the fractional $p(\cdot . \cdot)$-Laplacian operator in $\mathbb{R}^{N}$. Collect. Math. 72, 129-156 (2021)

5. Azroul, E., Benkirane, A., Shimi, M., Srati, M.: On a class of fractional $p(x)$-Kirchhoff type problems. Appl. Anal. 100, 383-402 (2021)

6. Bahrouni, A.: Comparison and sub-supersolution principles for the fractional $p(x)$-Laplacian. J. Math. Anal. Appl. 458 1363-1372 (2018)

7. Bahrouni, A., Radulescu, V.D.: On a new fractional Sobolev space and applications to nonlocal variational problems with variable exponent. Discrete Contin. Dyn. Syst., Ser. S 11(3), 379-389 (2018)

8. Benkhira, E.-H., Essoufi, E.-H., Fakhar, R.: On convergence of the penalty method for a static unilateral contact problem with nonlocal friction in electro-elasticity. Eur. J. Appl. Math. 27, 1-22 (2016)

9. Benslimane, O., Aberqi, A., Bennouna, J.: The existence and uniqueness of an entropy solution to unilateral Orlicz anisotropic equations in an unbounded domain. Axioms 9, 109 (2020)

10. Benslimane, O., Aberqi, A., Bennouna, J.: On some nonlinear anisotropic elliptic equations in anisotropic Orlicz space. Arab J. Math. Sci. (2020). https://doi.org/10.1108/AJMS-12-2020-0133

11. Benslimane, O., Aberqi, A., Bennouna, J.: Existence and uniqueness of entropy solution of a nonlinear elliptic equation in anisotropic Sobolev-Orlicz space. Rend. Circ. Mat. Palermo, II. Ser. 70, 1579-1608 (2021). https://doi.org/10.1007/s12215-020-00577-4

12. Benslimane, O., Aberqi, A., Bennouna, J.: Existence and uniqueness of weak solution of $p(x)$-Laplacian in Sobolev spaces with variable exponents in complete manifolds. Filomat 35, 1453-1463 (2021) https://doi.org/10.2298/FIL2105453B

13. Benslimane, O., Aberqi, A., Bennouna, J.: Existence results for double phase obstacle problems with variable exponents. J. Elliptic Parabolic Equ. 7, 875-890 (2021). https://doi.org/10.1007/s41808-021-00122-z

14. Biswas, R., Tiwari, S.: Variable order nonlocal Choquard problem with variable exponents. Complex Var. Elliptic Equ. 66 853-875 (2021)

15. Brezis, H.: Functional Analysis, Sobolev Spaces and Partial Differential Equations. Universitext. Springer, New York (2011)

16. Chen, S.T., Tang, X.H.: On the planar Schrödinger-Poisson system with the axially symmetric potential. J. Differ. Equ. 268, 945-976 (2020)

17. Chen, W., Rădulescu, V.D., Zhang, B.: Fractional Choquard-Kirchhoff problems with critical nonlinearity and Hardy potential. Anal. Math. Phys. 11, 1-25 (2021)

18. Chen, Y., Levine, S., Rao, M.: Variable exponent, linear growth functionals in image restoration. SIAM J. Appl. Math. 66, 1383-1406 (2006)

19. Chung, N.T., Ho, K.: On a p(.)-biharmonic problem of Kirchhoff type involving critical growth. Appl. Anal.. https://doi.org/10.1080/00036811.2021.1903445

20. Di Nezza, E., Palatucci, G., Valdinoci, E.: Hitchhiker's guide to the fractional Sobolev spaces. Bull. Sci. Math. 136, 521-573 (2012)

21. Fan, X., Zhao, D.: On the spaces $L^{p(x)}(\Omega)$ and $W^{m, p(x)}(\Omega)$. J. Math. Anal. Appl. 263, 424-446 (2001) 
22. Fan, X., Zhao, Y., Zhao, D.: Compact imbedding theorems with symmetry of Strauss-Lions type for the space $W^{1, p(x)}(\Omega)$. J. Math. Anal. Appl. 255, 333-348 (2001)

23. Fu, Y: The principle of concentration compactness in $L^{p(x)}$ spaces and its application. Nonlinear Anal., Theory Methods Appl. 71, 1876-1892 (2009)

24. Fu, Y., Guo, L.: Variable exponent spaces of differential forms on Riemannian manifold. J. Funct. Spaces Appl. 2012, Article ID 575819 (2012)

25. Gaczkowski, M., Górka, P.: Sobolev spaces with variable exponents on Riemannian manifolds. Nonlinear Anal., Theory Methods Appl. 92, 47-59 (2013)

26. Gaczkowski, M., Górka, P., Pons, D.J.: Sobolev spaces with variable exponents on complete manifolds. J. Funct. Anal. 270, 1379-1415 (2016)

27. Goodrich, C.S., Ragusa, M.A., Scapellato, A.: Partial regularity of solutions to $p(x)$-Laplacian PDEs with discontinuous coefficients. J. Differ. Equ. 268, 5440-5468 (2020)

28. Guo, L.: The Dirichlet problems for nonlinear elliptic equations with variable exponents on Riemannian manifolds. J. Appl. Anal. Comput. 5, 562-569 (2015)

29. Guo, L., Zhang, B., Zhang, Y.: Fractional p-Laplacian equations on Riemannian manifolds. Electron. J. Differ. Equ. 2018, 1 (2018)

30. Hebey, E.: Nonlinear Analysis on Manifolds: Sobolev Spaces and Inequalities: Sobolev Spaces and Inequalities, vol. 5. Am. Math. Soc., Providence (2000)

31. Ho, K., Kim, Y.: A-priori bounds and multiplicity of solutions for nonlinear elliptic problems involving the fractional p(.)-Laplacian. Nonlinear Anal. 188, 179-201 (2019)

32. Kaufmann, U., Rossi, J.D., Vidal, R.: Fractional Sobolev spaces with variable exponents and fractional $p(x)$-Laplacians. Electron. J. Qual. Theory Differ. Equ. 2017, Article ID 76 (2017)

33. Liu, H., Fu, Y.: Embedding theorems for variable exponent fractional Sobolev spaces and an application. AIMS Math. 6 , 9835-9858 (2021)

34. Rădulescu, V.D., Repovš, D.D.: Partial Differential Equations with Variable Exponents: Variational Methods and Qualitative Analysis. CRC Press, Boca Raton (2015)

35. Ragusa, M.A., Tachikawa, A.: Partial regularity of the minimizers of quadratic functionals with VMO coefficients. J. Lond. Math. Soc. 72, 609-620 (2005)

36. Ragusa, M.A., Tachikawa, A.: Regularity for minimizers for functionals of double phase with variable exponents. Adv. Nonlinear Anal. 9, 710-728 (2019)

37. Růžička, M.: Modeling, mathematical and numerical analysis of electrorheological fluids. Appl. Math. 49, 565-609 (2004)

38. Samko, S.: On a progress in the theory of Lebesgue spaces with variable exponent: maximal and singular operators. Integral Transforms Spec. Funct. 16, 461-482 (2005)

39. Tang, X.H., Chen, S.T.: Ground state solutions of Nehari-Pohozaev type for Kirchhoff-type problems with general potentials. Calc. Var. Partial Differ. Equ. 56, 110 (2017)

40. Tang, X.H., Cheng, B.T.: Ground state sign-changing solutions for Kirchhoff type problems in bounded domains. J. Differ. Equ. 261, 2384-2402 (2016)

41. Vázquez, J.L.: Growing solutions of the fractional $p$-Laplacian equation in the fast diffusion range. Nonlinear Anal. 214 $112575(2022)$

42. Vetro, C.: Variable exponent $p(x)$-Kirchhoff type problem with convection. J. Math. Anal. Appl. 506(2), Article ID $125721(2021)$

\section{Submit your manuscript to a SpringerOpen ${ }^{\circ}$ journal and benefit from:}

- Convenient online submission

- Rigorous peer review

- Open access: articles freely available online

- High visibility within the field

- Retaining the copyright to your article

Submit your next manuscript at $\boldsymbol{~ s p r i n g e r o p e n . c o m ~}$ 